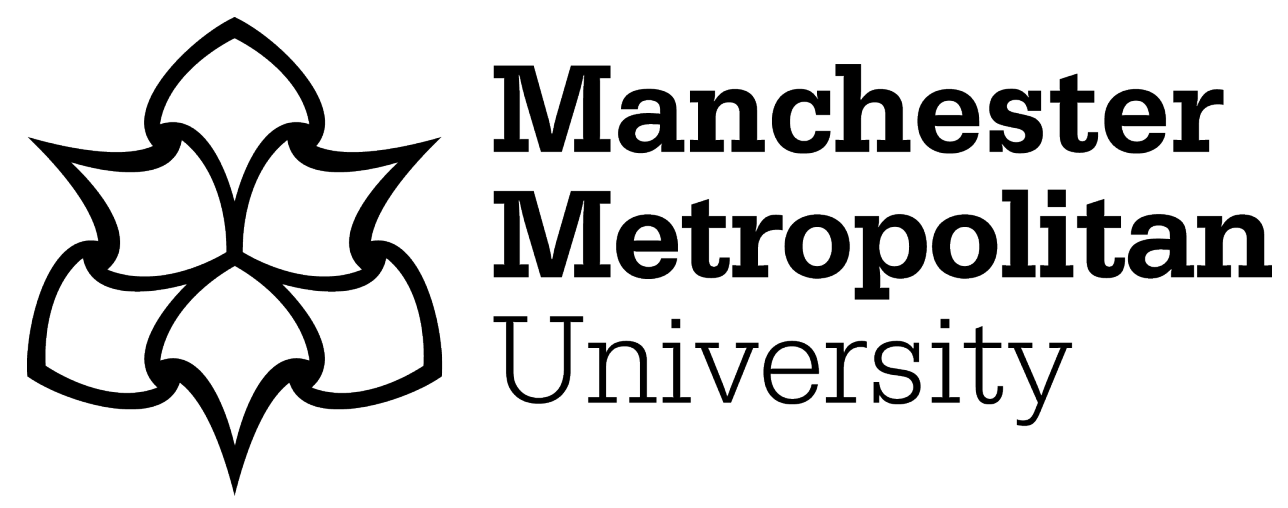

Chang, Y-H, Wang, W, Siebert, T, Chang, J-Y and Mottershead, JE (2018) Basis-updating for data compression of displacement maps from dynamic DIC measurements. Mechanical Systems and Signal Processing, 115. pp. 405-417. ISSN 0888-3270

Downloaded from: https://e-space.mmu.ac.uk/620834/

Publisher: Elsevier

DOI: https://doi.org/10.1016/j.ymssp.2018.05.058

Usage rights: Creative Commons: Attribution-Noncommercial-No Derivative Works 4.0

Please cite the published version 


\title{
Basis-updating for Data Compression of Displacement Maps from Dynamic DIC Measurements
}

\author{
Yen-Hao Chang ${ }^{\mathrm{a}, \mathrm{b}}$, Weizhuo Wang ${ }^{\mathrm{c}}$, Thorsten Siebert ${ }^{\mathrm{d}}$, Jen-Yuan Chang ${ }^{\mathrm{b}}$ and \\ John E. Mottershead ${ }^{\mathrm{a}^{*}}$ \\ ${ }^{a}$ Department of Mechanical, Aerospace and Materials Engineering, University of Liverpool, UK \\ ${ }^{b}$ Department of Power Mechanical Engineering, National Tsing Hua University, Taiwan \\ ${ }^{c}$ School of Engineering, Manchester Metropolitan University, UK \\ ${ }^{d}$ Dantec Dynamics GmbH, Kaessbohrer Str. 18, D 89077 Ulm, Germany
}

\begin{abstract}
The extraction of useful information and removal of redundant noise from data has become a major research topic in recent years. Data compression is necessary for all kinds of analysis, and the demand for efficient compression techniques has gained much attention. Digital image correlation is a camera-based measuring system, which has been widely applied in strain analysis because of the convenience of measuring displacement fields by simply selecting a region of interest. Currently, there is interest in applying such methods to engineering structures in dynamics. However, one of the major issues related to the integration of camera-based systems with dynamic measurement is the generation of huge amounts of data, typically extending to many thousands of data points, because of the requirements of high sampling rate, spatial resolution, and long duration of recording. In this paper a new algorithm is presented that addresses the need for efficiency in full-field data processing. By making use of the data itself and combining the concept of sparse representation with Gram-Schmidt orthogonalisation, the number of basis function used to represent the data can be reduced and a concise decomposition established. In both simulated and experimental cases, the compression ratios for data size and number of signals used in operational modal analysis are substantially diminished, thereby demonstrating the effectiveness of the proposed algorithm. A reduced number of new basis functions is determined for the representation of data under the condition that the reconstructed displacement map reproduces the raw measured data to within a chosen threshold on the coefficient of correlation.
\end{abstract}

Keywords: Shape descriptor, Dictionary updating, K-SVD, Sparse representation, Kernel function, Operational modal analysis

\section{Introduction}

With the ever-growing size of data, the problems of processing and storage have greatly increased to such an extent that data cannot be processed by personal computers with limited computational capacities directly. The extraction of useful information and removal of redundant data has become a major research topic in recent years. Data compression is now seen to be a necessity for analysis, and the demand for efficient compression techniques has gained the attention of numerous researchers. A particular aspect of this phenomenon occurs in the processing of large volumes of full-field vibration data by photogrammetry. In an extensive review, extending to some 130 citations, Baqersad et al. [1] listed four main approaches to dynamic (vibration) measurement, namely the conventional point-wise sensor measurement (e.g. Ewins [2]), interferometry, laser Doppler vibrometry and photogrammetry. The advantages of photogrammetry over other methods was said to include the ease of extracting rigid body modes and its suitability for highly-flexible or rotating structures. Further sub-classification of photogrammetry included point tracking, digital image correlation (DIC) and target-less approaches, with DIC [3] being the most widely applied. In a

\footnotetext{
* Corresponding author: John E. Mottershead, Department of Mechanical, Aerospace and Materials Engineering, University of Liverpool, Harrison-Hughes Building, The Quadrangle, Liverpool L69 3GH, UK.

E-mail address: j.e.mottershead@liv.ac.uk (J. E. Mottershead).
} 
review of 3D high-speed DIC for vibration measurement, Beberniss and Ehrhardt [4] pointed to a difficulty at high frequency because of low out-of-plane sensitivity. This was overcome separately by Poozesh et al. [5] and Molina-Viedma et al. [6] using phase-based motion magnification and results are reported in [7] for 3D operational deflection shapes in the range from $1500 \mathrm{~Hz}$ to $6710 \mathrm{~Hz}$ without the need for high levels of excitation. Poozesh et al. [8] and Patil et al. [9] developed methods for the stitching of DIC images of sub-areas of very large structures to obtain complete images of vibration modes. Javh et al. [10] developed an efficient gradient-based optical flow method and reported displacement resolutions of less than a thousandth of a pixel. They demonstrated the application of their method on the vibration modes of a cymbal up to $2373 \mathrm{~Hz}$. The numerous applications include the displacement measurement of the blade of a toy helicopter using DIC by Sousa et al. [11], model updating of hypersonic aircraft panels by Perez et al. [12] and low-velocity impact on composite plates by Flores et al. [13]. However, one of the major issues related to the integration of camera-based systems and dynamic measurement is the generation of huge amount of data, often extending to several thousands of data points, because of the requirement of high sampling rate up to the Nyquist frequency, spatial resolution related to number of pixels in images, and frequency resolution requiring long durations of recording.

One of the difficulties of using data from photogrammetry is its comparison with finite element (FE) predictions, since DIC grids are different from FE nodes. Leclerc et al. [14] directly compared the measured displacement field with simulated numerical results and implemented model updating by minimizing the difference between them. Other than the direct comparison, Wang et al. [15-19] and Patki and Patterson [20] developed image processing and pattern recognition techniques capable of extracting important features from displacement fields through predetermined kernel functions, such as geometric polynomials [21], Zernike [22], Tchebichef [23], or Krawtchouk [24] polynomials or wavelets [25]. With the use of such basis functions (or kernels), large amount of full-field data may be represented by just a few shape descriptor (SD) terms (also called moments) with high-fidelity. Each type of kernel functions is suitable for a particular geometric domain. For example, the Zernike polynomials are widely applied on domains with circular geometry, and Tchebichef polynomials on rectangular domains. Wang et al. [26] developed a technique known as the adaptive geometric moment descriptor (AGMD) to deal with the issue of selecting kernels for different domains with three-dimensional irregular geometry and holes. They carried out modal tests on a car bonnet liner entirely in the SD domain and reconstructed the first eleven mode shapes in the physical domain from the SD contributions.

For computer scientists in the field of image processing, the terminology used to describe decomposition is different from that used in structural dynamics. For example, basis functions or kernels are known as dictionaries, and an atom or word describes an element contained in a dictionary. Current research on decomposing images with overcomplete dictionaries (i.e. more atoms than the dimension of image) has applications that benefit from sparsity of representation, such as image denoising [27, 28], single image super-resolution reconstruction [29, 30], image inpainting [31, 32], and image compression [33]. A review of sparse representation in dictionary learning and image fusion (by using multiple sensors) was carried out by Zhang et al. [34]. One of the most cited algorithms, K-SVD, proposed by Aharon et al. [35] is a method for updating an initial dictionary with targeted data. After updating, the representation of the data will become sparser but still satisfying the representational-error requirement. This algorithm is inspired by K-means (also known as the Lloyd-Forgy method [36]) and the dictionary learning method proposed by Lesage et al. [37]. The well-known compressed sensing, proposed by Donoho [38], is based upon the concept of sparse representation and suggests that the required number of samples for reconstruction under mild conditions can be fewer than that predicted by the Nyquist-Shannon theorem.

The motive of the research is that AGMD as proposed by Wang et al. [26] might not provide the best decomposition of a full-field image, because the data are not considered in the generation of the basis. In this paper, an updating algorithm for the basis or dictionary, which is utilised to decompose huge amounts of raw data, is proposed, and a comparison of the results from AGMD and the new method is made on real engineering structures. Within a finite number of iterations, a more succinct or representative description of data is generated, i.e. a fewer number of SD terms than the number of AGMD terms containing the same amount of information. The mathematical details of AGMD are reviewed in $\S 2$ and the proposed basis-updating algorithm is presented in $\S 3$. $\$ 4$ briefly explains some of the details of a number of Operational Modal Analysis (OMA) methods to be applied in later sections and illustrates the analysis procedures in a flow chart. Two data sets, simulated and experimental, are described in $\S 5$. The results of identification with AGMD signals and those generated from the proposed method are shown and discussed in $\$ 6$. Conclusions are drawn in $\$ 7$. 


\section{Initial basis and shape representation}

There are several different kernel functions available for the decomposition of shapes. Of these, the AGMD [26] is suitable for any geometry or discontinuity and is based upon geometric monomials [21]. Both are presented briefly in this section with mathematical formulae and examples.

\subsection{Geometric monomials}

The idea of using geometric monomials for visual pattern recognition and the computation of corresponding geometric moments was first proposed by Hu [21]. The main advantage of the geometric moment is its invariance in respect to properties, such as rotation, translation, and scale. The geometric monomials may be written as,

$$
G_{p q}=x^{p} y^{q}
$$

where $x$ and $y$ are coordinates, and $p$ and $q$ are monomial orders.

\subsection{Adaptive geometric moment descriptor (AGMD)}

Wang et al. [26] proposed the AGMD as a solution to the problem of finding basis function applicable to general shapes as encountered in engineering. For curved surfaces, it is firstly necessary to carry out an isomorphic mapping from the 3D surface to a 2D plane. In [26], this was achieved by bijectively using discrete conformal mapping as described by Desbrun et al. in [39]. This process involves meshing of the surface so that the difference in angle of individual triangular-element vertices on the curved surface and flat plane is minimized (Pinkall and Polthier in [40]). To establish a compact decomposition, Wang et al. [18] proposed an orthonormal basis achieved using Gram-Schmidt orthonormalisation (GSO).

Before the explanation of GSO, the definition of inner product is first given. The inner product of two arbitrary functions $u$ and $v$ in a continuous domain $\Omega(\chi)$ is defined by,

$$
\langle u, v\rangle=\int_{\Omega(\chi)} u(\chi) v(\chi) d \chi
$$

and the orthogonality of the functions is confirmed when the inner product is zero.

For a discrete domain, the inner product of two arbitrary functions $u$ and $v$ can be represented by the discrete version of Eq. (2),

$$
\langle u, v\rangle=\sum_{i} u\left(\chi_{i}\right) v\left(\chi_{i}\right) d \chi_{i}
$$

The GSO of a set of kernel functions $\left\{Q_{1}, Q_{2}, \ldots, Q_{n}\right\}$ in a discrete domain may be expressed as,

$$
\left\{\begin{array}{c}
R_{1}=Q_{1} \\
R_{k}=Q_{k}-\sum_{r=1}^{k-1} \frac{\left\langle R_{r}, Q_{k}\right\rangle}{\left\langle R_{r}, R_{r}\right\rangle} R_{r}, k=2, \ldots, n
\end{array}\right.
$$

where $\left\{R_{1}, R_{2}, \ldots, R_{n}\right\}$ is an orthonormal set of kernel functions.

By the procedures explained herein, an orthonormal basis may be formed for any 3D surface. In Fig. 1 and Fig. 2, a comparison of geometric monomials and the polynomials generated from AGMD is illustrated. 


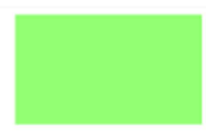

$(0,0)$

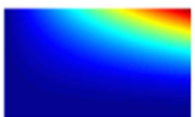

$(1,2)$

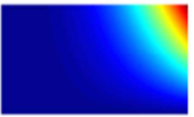

$(3,1)$

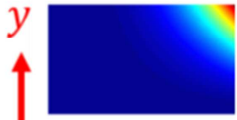

$(4,2)$

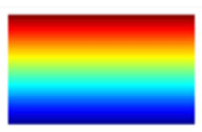

$(0,1)$

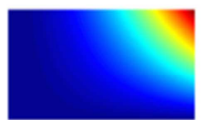

$(2,1)$

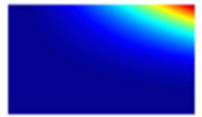

$(2,3)$

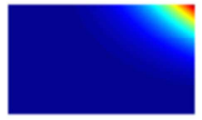

$(4,3)$

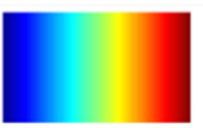

$(1,0)$

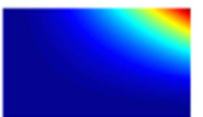

$(2,2)$

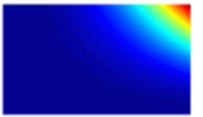

$(3,2)$

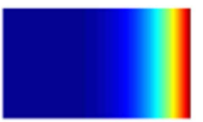

$(5,0)$

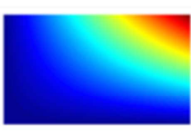

$(1,1)$

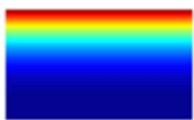

$(0,3)$

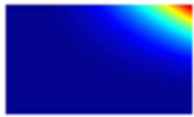

$(3,3)$

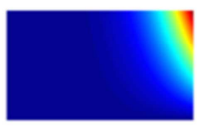

$(5,1)$

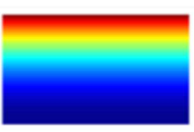

$(0,2)$

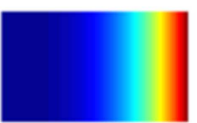

$(3,0)$

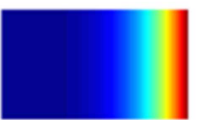

$(4,0)$

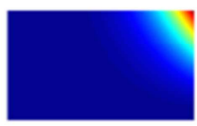

$(5,2)$

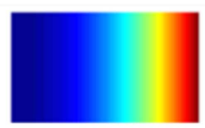

$(2,0)$

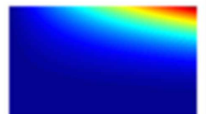

$(1,3)$

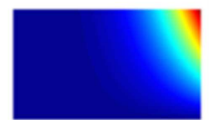

$(4,1)$

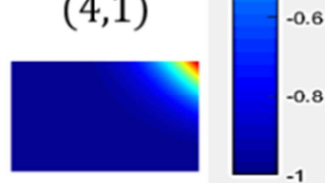

$(5,3)$

Fig. 1. First 24 scaled geometric monomials with orders in $\mathrm{x}$ and $\mathrm{y}$ directions respectively.

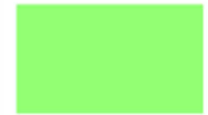

$(0,0)$

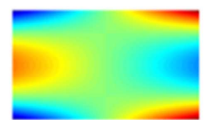

$(1,2)$

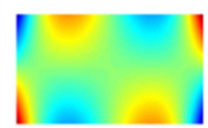

$(3,1)$

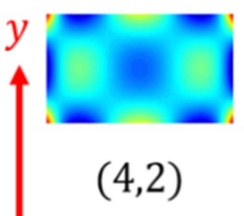

$(2,3)$

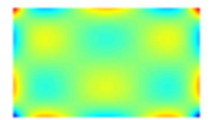

$(4,3)$

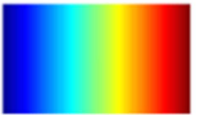

$(1,0)$

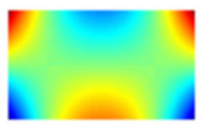

$(2,1)$
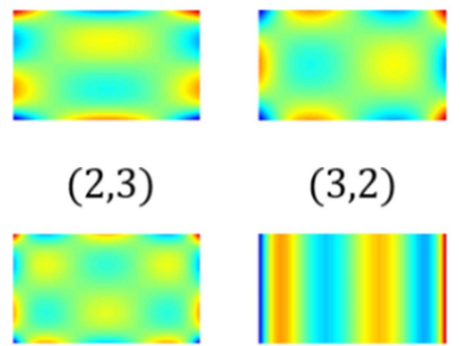

$(5,0)$

$(2,2)$

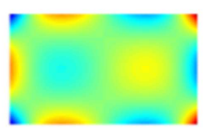

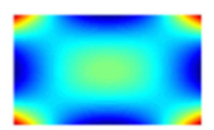

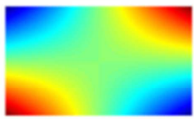

$(1,1)$

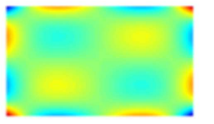

$(3,3)$

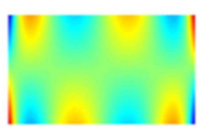

$(5,1)$

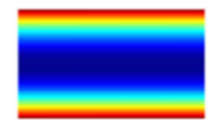

$(0,2)$
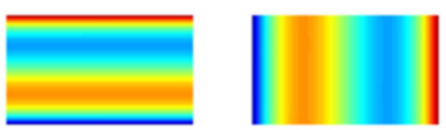

$(3,0)$

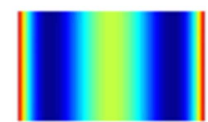

$(4,0)$

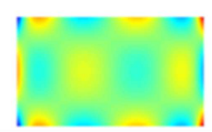

$(5,2)$

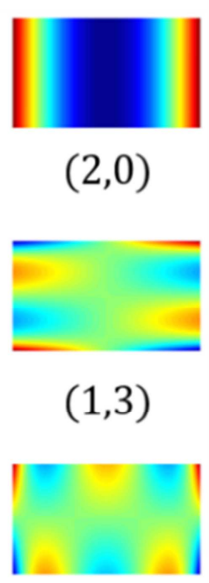

$(4,1)$

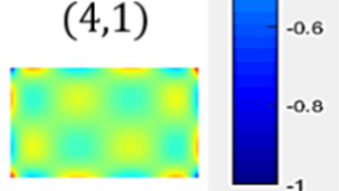

$(5,3)$

Fig. 2. First 24 scaled polynomials by AGMD with orders in $\mathrm{x}$ and $\mathrm{y}$ directions respectively. 


\subsection{Shape representation}

The shape descriptor, or coefficient, of a displacement map $W$, as well as the reconstructed displacement $\widehat{W}$, may be written together as,

$$
\left\{\begin{array}{c}
S D_{i}=\frac{\left.<\mathrm{W}, S_{i}\right\rangle}{\left.<S_{i}, S_{i}\right\rangle} \\
\widehat{W}=\sum_{i} S D_{i} \cdot S_{i}
\end{array}\right.
$$

where $i, S D_{i}$ and $S_{i}$ are the index of polynomial, the $i$-th shape descriptor and the $i$-th polynomial, respectively.

\section{Basis-updating algorithm}

As can be seen from the procedure described above, the formulation of the AGMD is done completely independently of the displacement map that it seeks to reproduce. Hence, by taking inspiration from the K-SVD algorithm as proposed by Aharon et al. in [35] and the utilisation of GSO, a new algorithm for basis-updating for DIC displacement maps is proposed herein. The procedure of the algorithm is described below:

1. Generate an initial basis (dictionary) $A=\left[A_{1}, \ldots, A_{m}\right]$ representing data with corresponding coefficients $S=\left[S_{1}, \ldots, S_{m}\right]$ and set a tolerance $\epsilon$ on the representation error.

2. Sequentially update each kernel function (atom) with data $X$ :

(i) Calculate the complement $X_{k}(1 \leq k \leq m)$,

$$
X_{k}=X-\sum_{j \neq k} A_{j} S_{j}
$$

(ii) Apply singular value decomposition on $X_{k}$,

$$
X_{k}=U S V^{T}
$$

(iii) Update $A_{k}$ using the first column of $U$,

$$
A_{k}=U_{1}
$$

3. Apply the GSO process to updated $A=\left[A_{1}, \ldots, A_{m}\right]$ to ensure orthogonality and remove repeated kernel functions.

4. Calculate the updated coefficients $S=\left[S_{1}, \ldots, S_{m}\right]$.

5. Sort $S$ and $A$ according to the significance of each $S_{k}$.

6. If $\|X-A S\|>\epsilon$, go to step 2, else remove the most insignificant coefficient and corresponding shape and go to step 2 .

7. Repeat steps 2 to 6 until convergence is achieved.

In step 5 , the significance of each $\mathrm{SD}$ term $\left(S D_{i}, i=1,2, \ldots\right)$ is determined according to its absolute value in descending order. Thus, the kernel function that is most represented in the displacement map is considered to be the most significant. In what follows the algorithm described above is applied to operational (or output-only) modal data. 


\section{Operational modal analysis (OMA)}

Due to the generally high physical dimensions and comparatively low frequency range of civil structures, the identification of modal properties by conventional modal analysis techniques is a relatively troublesome task. The excitation of such structures cannot be easily implemented and usually demands specially designed costly devices for the generation of programmable and measurable artificial forces. In response to this problem, researchers in civil engineering have turned to OMA. Relative to the traditional input-output techniques, OMA makes good use of the obtainable ambient excitations as input and determines modal properties (natural frequencies, mode shapes, modal damping etc.) from measured responses with the assumption that the excitation has constant spectral density in the form of white noise, which is often approximately true for natural excitations, such as wind gusts and waves. Hence, OMA has become an established procedure for modal testing in civil engineering. For mechanical engineers, OMA can also serve as an alternative method for modal identification. There are two classes of OMA methods, i.e. the frequency-domain and time-domain techniques.

\subsection{Time-domain method}

Temporal signals are directly processed when using time-domain OMA methods, which may be separated into three main approaches as: stochastic subspace identification (SSI) [41], random decrement Ibrahim time domain (RD-ITD) method [42-44], and blind source separation (BSS) [45].

RD-ITD and BSS are not included in the present discussion, because of the requirement for an additional fitting procedure to identify the modal parameters. Peeters and De Roeck [41] described the first step of the SSI method as the construction of a Hankel matrix composed of measured data. The main difference between SSI-COV and SSI-Data is how the data is processed, i.e. the covariance or projection matrix between 'future' and 'past' parts of the Hankel matrix. Both methods apply singular value decomposition (SVD) on the covariance or projection matrix to establish a matrix of observability, from which the system matrix may be determined using conventional control theory.

By altering the dimensions of observability matrix, the dimensions of system matrix changes, and a stabilisation diagram can be formed by plotting all the poles for system matrices of different dimensions. From the stabilisation diagram and with the help of clustering techniques, such as hierarchical clustering as described in [46], stable poles, which corresponds to modes, may be selected.

The mode shapes found by SSI have complex values and may be converted to real mode shapes [47]. When working in the SD domain, real mode shapes in the spatial domain may be obtained by the linear combination of kernel functions and corresponding SDs.

\subsection{Frequency-domain method}

Frequency-domain methods make use of the matrix of output auto- and cross-spectral densities. There are four main types of frequency-domain method: frequency domain decomposition (FDD) [48], the transmissibility function method [49, 50], poly-reference least-squares complex frequency domain (P-LSCF) method [51], and Bayesian modal identification [52-54].

In this section, the details of three different frequency-domain methods are briefly explained, and they are FDD, Bayesian, and P-LSCF methods. The Bayesian approach is based upon the first singular value spectrum calculated from FDD, which is included for that reason, even though additional SDOF curve fitting is necessary.

Before the application of frequency-domain methods, an extra discrete Fourier transform (DFT) step is necessary. A 3D matrix of frequency spectra is formed, where each 2D submatrix contains auto- and cross- spectra at a specific frequency and the third dimension corresponds to the discrete frequency points of the DFT. By applying SVD on each 2D submatrix, the envelope of the sum of all first singular value spectra is formed. From this spectrum, the potential modes of system may be selected by peak-picking (PP).

The identification of modes by the Bayesian method starts with the choice of peaks and frequency ranges of the singular value spectrum. Optimisation is based upon the gradient descent, and the modal parameters are identified by minimising the negative log of an objective function. Other than the selection of initial conditions, a convergence condition is also selected by the user.

In the P-LSCF method, the determination of modes is similar to SSI, which relies on the stabilisation diagram and hierarchical clustering. However, the main difference is the computation of system poles, which depend upon the system matrices formed by a right matrix fraction. User-chosen parameters are the length of each time segment and the 
time lag between segments. The longer the time segment and time lag, the more the frequency lines and fewer the segments, and vice versa.

Applications of OMA methods are described by numerous authors, including Oliveira et al. [55] who reported on the continuous monitoring of an onshore wind turbine using the SSI-COV and P-LSCF methods, and Chen at al. [56] who used the SSI-Data and FDD techniques in the OMA of a multi-span concrete bridge. The ESA Vega launcher was investigated using OMA by De Vivo et al [57], and Chomette and Carrou [58] studied the modes of a concert harp. Very recent developments include uncertainty quantification applied to the SSI-COV approach by Reynders et al. [59], and development of the Bayesian approach to account for asynchronous measurements from multiple set-ups by Zhu et al. [60-62]. A combination of DIC method and OMA was presented in [63]. The first investigation and application of integrated SD and OMA methods on simulated data was provided by Chang et al. [64]. Analysis was carried out in the SD domain, with mode shapes finally converted to the physical (spatial) domain.

\subsection{Analysis procedure}

The analysis procedure is illustrated in the flowchart of Fig. 3, with steps explained as follows. In the first step, a collection of measured displacement maps serves as the target of the analysis. These maps are then decomposed into several SDs and kernel functions, generated by the AGMD method and further updated by the algorithm described in $\S 3$. After decomposition, the SD signals are used as input for OMA in the identification of natural frequencies, damping ratios, and complex mode shapes, and the mode shapes are further converted to real number as described previously. Since the analysis is implemented in SD domain, another step of conversion is necessary for the presentation of mode shapes in spatial domain. This is done by linear combination of mode shapes of SDs from step 2 and kernel functions from step 1 .

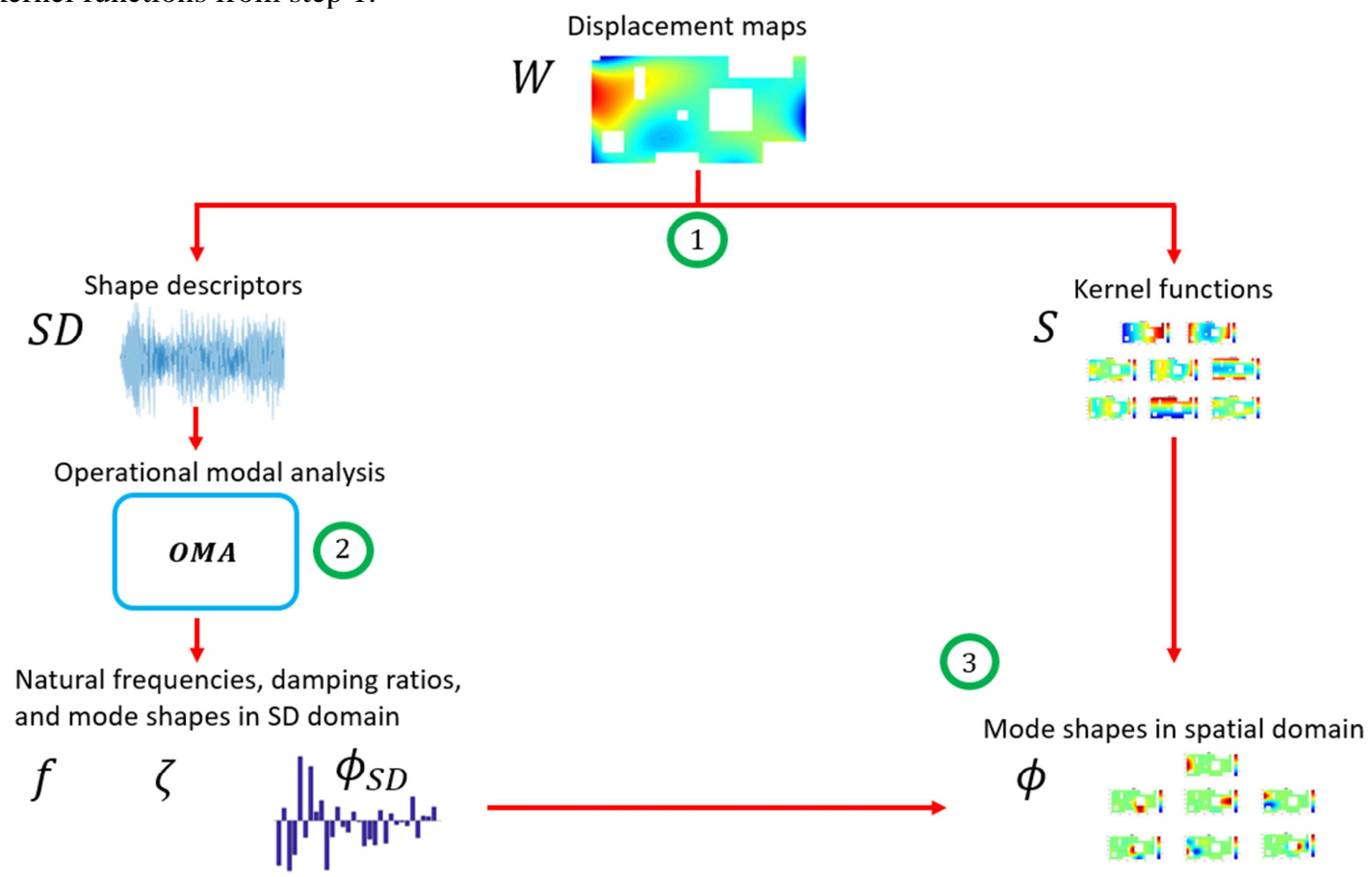

Fig. 3. Flow chart of analysis.

\section{Case Studies}

For the validation of the proposed algorithm, both simulated and experimental data are analysed. 


\subsection{Simulated data}

Simulated data is produced by using the ABAQUS FE code. The FE mesh of an irregular plate-like structure with fully-fixed and pinned boundary conditions is shown in Fig. 4. Full details of the FE model and analysis are provided in Table 1 and the first seven modal frequencies are given in Table 2.

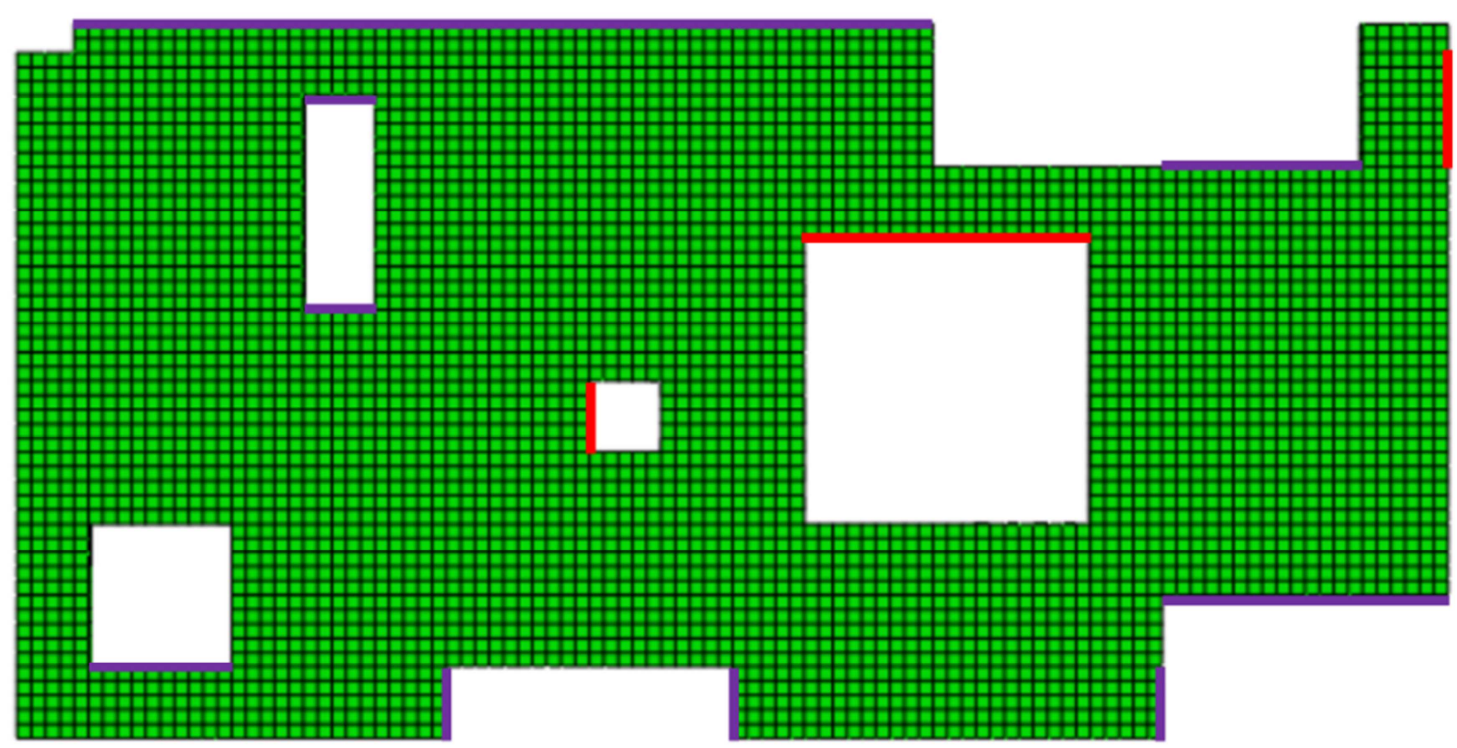

Fig. 4. FE model: Purple line - fixed in 6 degrees of freedom; Red line - pinned in 3 degrees of freedom.

Table 1. The parameters of FE simulation.

\begin{tabular}{|l|l|}
\hline Material & $\begin{array}{r}\text { A36 Steel: Young's Modulus: } 200 \mathrm{GPa} \\
\text { Poisson's ratio: } 0.26 \\
\text { Density: } 7850 \mathrm{Kg} / \mathrm{m}^{3}\end{array}$ \\
\hline Dimension & Width: 1 m Height: $0.5 \mathrm{~m}$ Thickness: $0.01 \mathrm{~m}$ \\
\hline Section & Solid, Homogeneous \\
\hline Element size & $0.01 \mathrm{~m}$ by 0.01 m by $0.01 \mathrm{~m}$ \\
\hline Boundary condition & As shown in Fig. 5 \\
\hline Excitation & Excitation: Zero-mean Gaussian white noise $(0 \sim 100 \mathrm{~Hz})$ \\
\hline Sampling frequency & $1000 \mathrm{~Hz}$ \\
\hline Number of steps & $4000(4$ seconds $)$ \\
\hline Output & 4044 out-of-plane displacement recordings \\
\hline
\end{tabular}

Table 2. Natural frequencies. (Hz)

\begin{tabular}{|l|l|l|l|l|l|l|l|}
\hline Mode & 1 & 2 & 3 & 4 & 5 & 6 & 7 \\
\hline FE & 35.783 & 54.863 & 63.342 & 71.012 & 82.858 & 89.328 & 94.546 \\
\hline
\end{tabular}

\subsection{Experimental PCB circuit board}

The circuit board is a real experimental example of DIC data from an industrial company. It is partly obscured by other parts so that the camera has only two separate parts of the circuit board in view, as shown in Fig. 5. The boundary condition of the circuit board was not known by the company, who were able to provide only the following information. The structure was excited by a random excitation with a frequency range from $200 \mathrm{to} 1000 \mathrm{~Hz}$, and the sampling frequency of the high-speed cameras was set at $2000 \mathrm{~Hz}$. The total duration of measurement was 1.579 
seconds, and hence the resulting number of steps was 3158 .

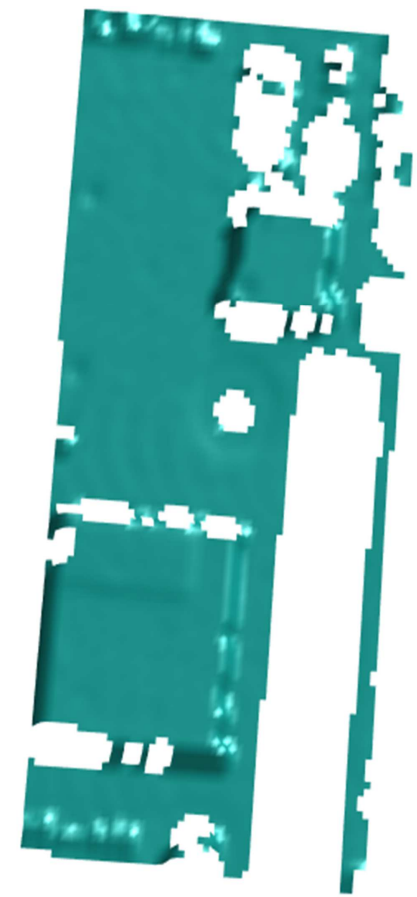

(a) part 1

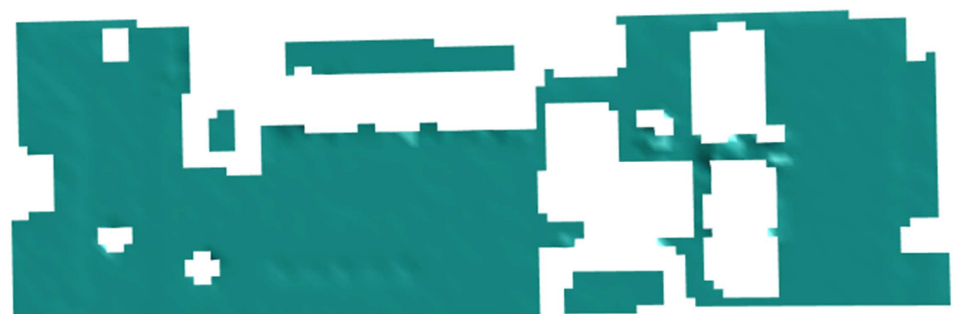

(b) part 2

Fig. 5. Circuit board parts 1 (a) and 2 (b).

\section{Result and discussion}

In this section, the results of OMA with AGMD and updated kernels in simulated and experimental cases are provided, compared, and discussed.

\subsection{Simulated data}

The algorithm in $\$ 3$ allows for a threshold to be set on the coefficient of correlation between the original data and its representation by using the updated basis. In the present example, the number of modes within the excitation range is 7, thus at least 7 kernel functions are necessary for a successful reconstruction of mode shapes. Fig. 6 shows that if the threshold is too low, then over-compression can happen, i.e. some of the mode shapes might not be successfully identified and reconstructed.

Fig. 7 shows the tables of modal assurance criteria (MAC) values between the original data and either 30 AGMD terms, 7 AGMD terms and 7 terms of the updated basis. The three rows of the figure correspond to 3 different OMA methods $\left(1^{\text {st }}\right.$ row: SSI-COV/ $2^{\text {nd }}$ row: P-LSCF/ $3^{\text {rd }}$ row: Bayesian). The MAC maps of mode shapes identified from each OMA method with updated SDs are better than those that include the 30 most significant terms AGMD kernels. Also, with the same number of kernel functions, some of the mode shapes were not identifiable using AGMDs, but the proposed method succeeded in the identification by preserving the important features of the displacement maps. Fig. 8 shows the effect of over-compression, when the number of kernel functions was less than the number of modes, so that the MAC matrix could not be diagonalised. For comparison, the compression ratios in terms of data size and number of shape functions are provided in Table 3, and as can be seen, both compression-ratio measures are significantly improved by the proposed method. 
Table 3: Compression ratios for simulated data.

\begin{tabular}{|l|l|l|l|l|}
\hline & Data size (bytes) & Compression ratio & Data number & Compression ratio \\
\hline Original & $115 \mathrm{MB}$ & none & 4044 data points & none \\
\hline AGMD & $1.76 \mathrm{MB}$ & 65.34 & 30 SD terms & 134.80 \\
\hline proposed method & $465 \mathrm{~KB}$ & 253.25 & 7 SD terms & 577.71 \\
\hline
\end{tabular}

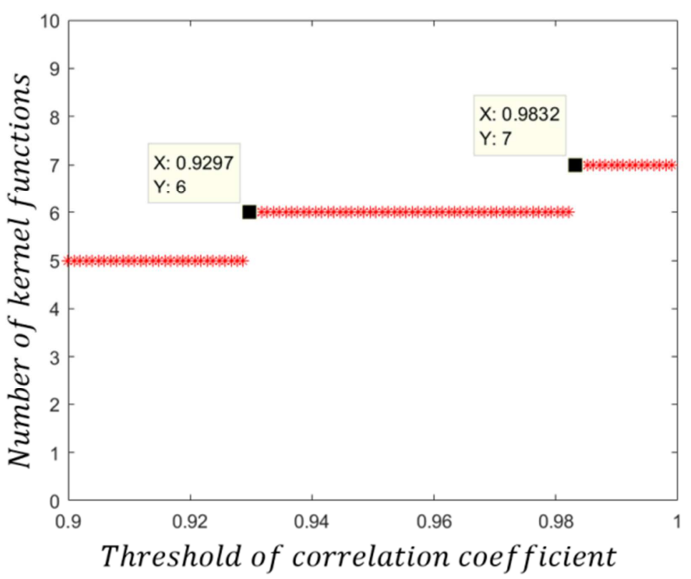

Fig. 6. Number of kernel functions vs. threshold of correlation coefficient.

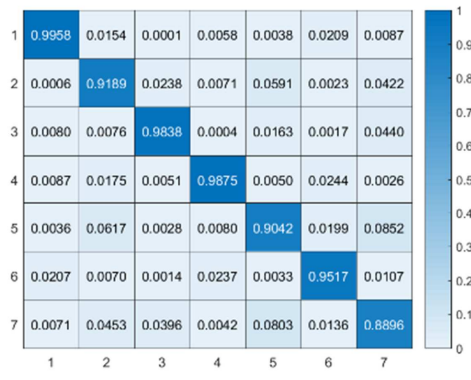

(a) 30 terms of AGMD

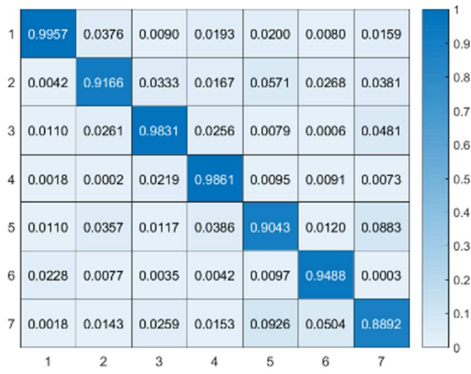

(d) 30 terms of AGMD

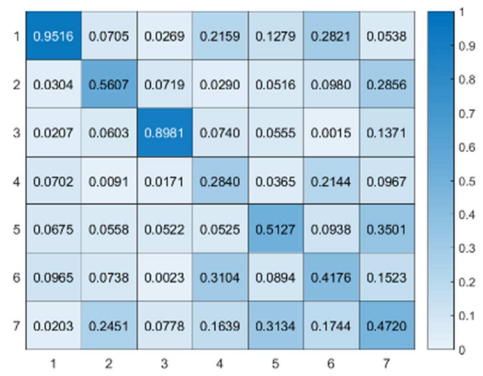

(b) 7 terms of AGMD

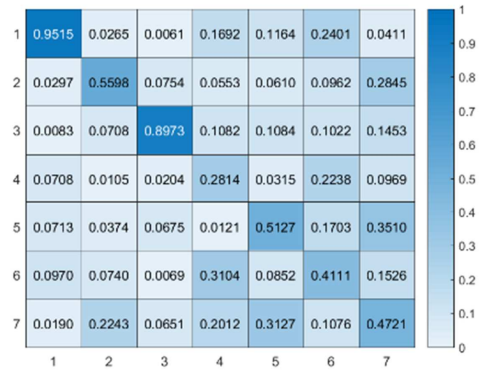

(e) 7 terms of AGMD

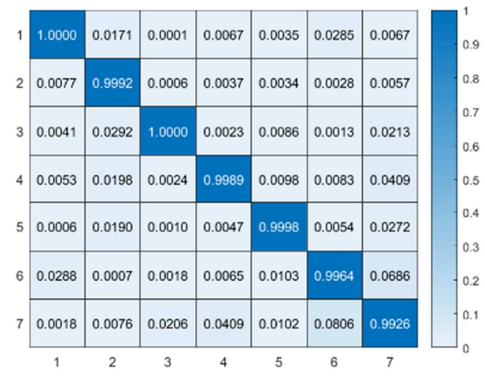

(c) Updated basis of 7 terms

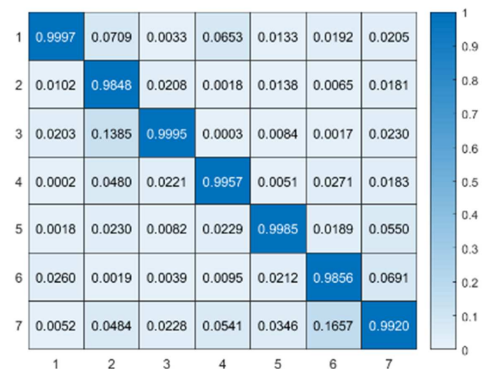

(f) Updated basis of 7 terms 


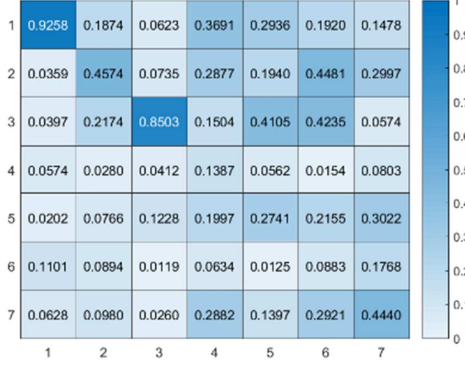

$\begin{array}{ll}\text { (g) } 30 \text { terms of AGMD } & \text { (h) } 7 \text { terms of AGMD } \\ \text { Fig. 7. MAC maps for AGMDs and updated SDs. First row: SSI }\end{array}$ method ( $\mathrm{x}$ axis - SD, y axis - FE).
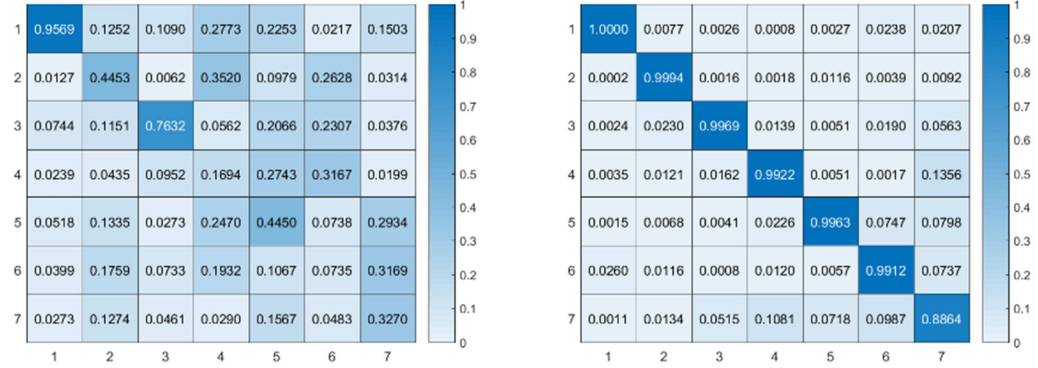

(i) Updated basis of 7 terms

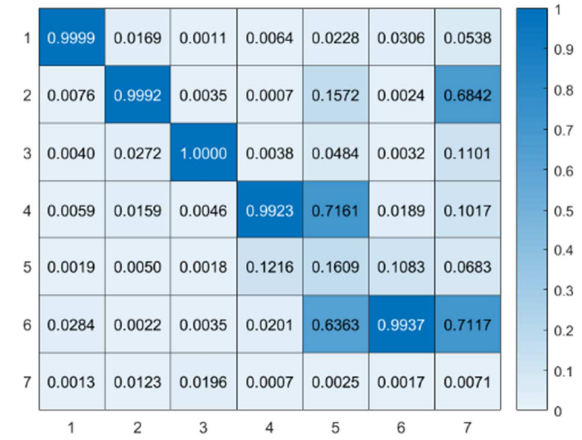

(a) Updated basis of 5 terms by SSI-COV

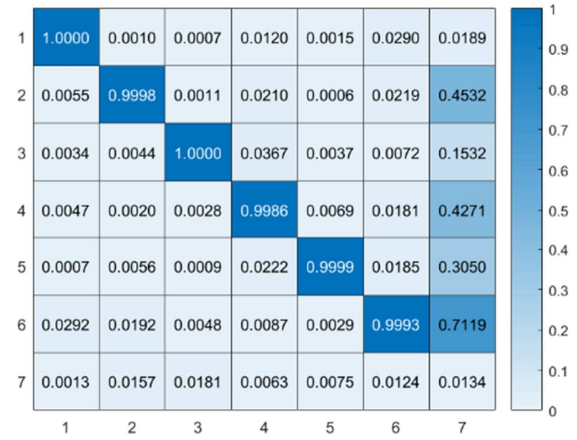

(b) Updated basis of 6 terms by SSI-COV

Fig. 8. MAC maps from over-compressed bases. (x axis - SD, y axis - FE,)

\subsection{Printed circuit board (PCB)}

For the experiment, the same procedures are applied to determine the modal properties, and the data from both parts of the circuit board are combined in the analysis, with the resulting identified natural frequencies provided in Table 4.

Table 4. Identified natural frequencies of circuit board by SSI-COV. (Hz)

\begin{tabular}{|l|l|l|}
\hline Mode & 1 & 2 \\
\hline 80 terms of AGMD & 534.8856 & 779.037 \\
\hline Updated basis of 8 terms & 535.297 & 779.0385 \\
\hline
\end{tabular}

Just two modes were identified in the excitation range, but because of the effects of noise and out-of-range modes the number of SDs required to reproduce the data was found to be greater than 2 . The compression ratios of both parts 1 and 2 of the circuit board are presented in Tables 5 and 6, where the advantages of the proposed method are apparent. It is seen in Fig. 9 that eight updated SDs are necessary to achieve a correlation coefficient of 0.99. Fig. 10 shows virtually no difference between 80 AGMD terms and 8 updated SD for the first mode, whereas slight differences can be seen in the second mode. However, when viewing the two videos provided in the supplementary information, showing the deflected shapes of the second part of the circuit board when excited by a sine wave at $537.3 \mathrm{~Hz}$ and 779.3 $\mathrm{Hz}$ respectively, it becomes apparent that the second mode shape from the updated basis is the correct one. 
Table 5. Compression ratios of circuit board part 1.

\begin{tabular}{|l|l|l|l|l|}
\hline & Data size (bytes) & Compression ratio & Data number & Compression ratio \\
\hline Original & $119 \mathrm{MB}$ & none & 5300 data points & none \\
\hline AGMD & $5.14 \mathrm{MB}$ & 23.15 & 80 SD terms & 66.25 \\
\hline proposed method & $745 \mathrm{~KB}$ & 163.57 & 8 SD terms & 662.5 \\
\hline
\end{tabular}

Table 6. Compression ratios of circuit board part 2.

\begin{tabular}{|l|l|l|l|l|}
\hline & Data size (bytes) & Compression ratio & Data number & Compression ratio \\
\hline Original & $51.8 \mathrm{MB}$ & none & 2791 data points & none \\
\hline AGMD & $3.58 \mathrm{MB}$ & 14.47 & 80 SD terms & 34.89 \\
\hline proposed method & $480 \mathrm{~KB}$ & 110.51 & 8 SD terms & 348.88 \\
\hline
\end{tabular}

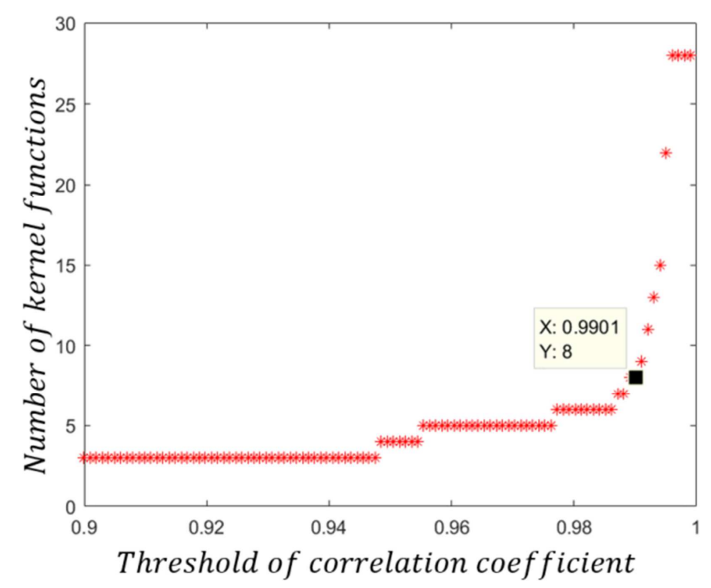

Fig. 9. Number of kernel functions vs. threshold of correlation coefficient.

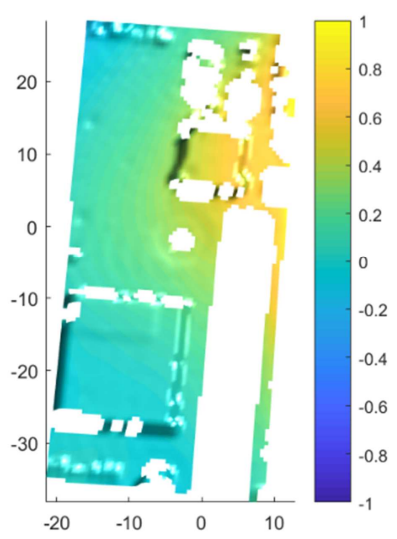

(a) First mode shape of circuit board part 1:80 terms of AGMD by SSI-COV

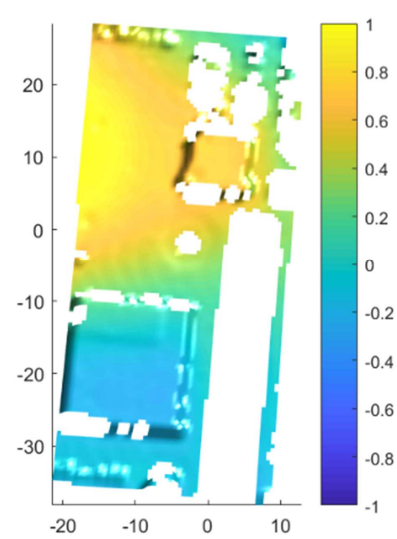

(b) Second mode shape of circuit board part 1: 80 terms of AGMD by SSI-COV 


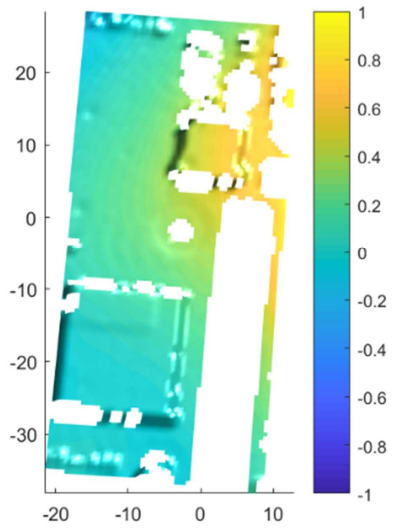

(c) First mode shape of circuit board part 1: updated basis of 8 terms by SSI-COV

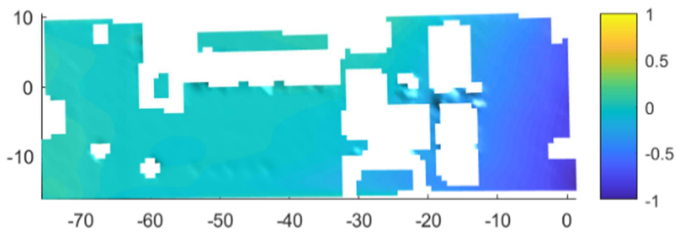

(e) First mode shape of circuit board part 2: 80 terms of AGMD by SSI-COV

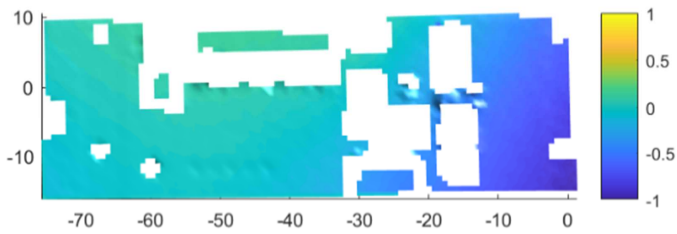

(g) First mode shape of circuit board part 2: updated basis of 8 terms by SSI-COV

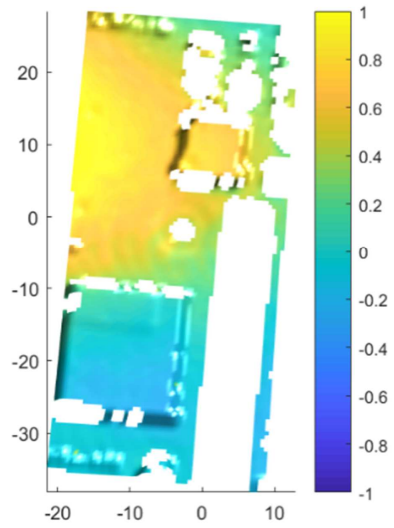

(d) Second mode shape of circuit board part 1: updated basis of 8 terms by SSI-COV

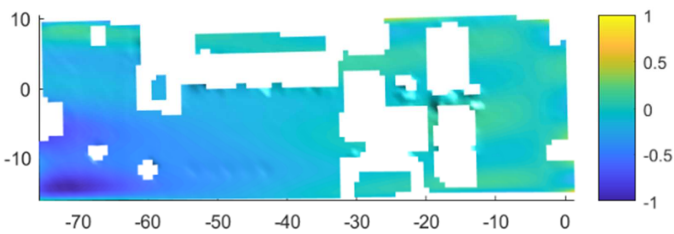

(f) Second mode shape of circuit board part 2: 80 terms of AGMD by SSI-COV

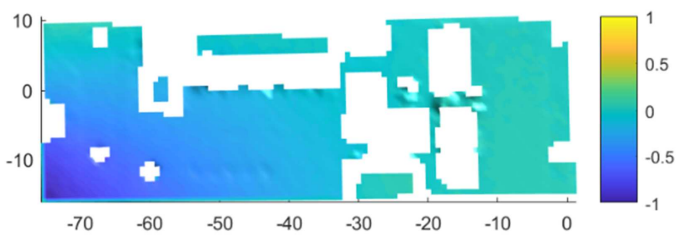

(h) Second mode shape of circuit board part 2: updated basis of 8 terms by SSI-COV

Fig. 10. The identified scaled mode shapes of both circuit board parts. (dimensions in $\mathrm{mm}$ )

\section{Conclusion}

A new algorithm has been presented for the compression of full-field measurements from DIC. It requires an initial basis as a starting point in an iterative procedure. Probably the most suitable initial basis is that provided by the AGMD, which is ideal for engineering structures and components, because it is capable of providing an orthogonal basis over an arbitrarily shaped domain. The working of the algorithm depends upon the data itself and it is this feature that enables it to achieve such significant improvements in compression ratio over classical orthogonal kernels (including the AGMD). It is particularly useful that a threshold may be placed on the correlation with the raw measured data to ensure that important features of DIC displacement maps are not lost by truncation. Validation of the algorithm has been carried out using both simulated datasets and physical measurement from a real industrial DIC investigation.

\section{Acknowledgement}

Yen-Hao Chang wishes to acknowledge the support provided by a University of Liverpool / NTHU scholarship 
through the dual PhD programme. DIC data from the PCB was kindly provided by Dantec Dynamics GmbH.

\section{References}

1. J. Baqersad, P. Poozesh, C. Niezrecki, P. Avitabile. 2017. Photogrammetry and optical methods in structural dynamics - a review, Mechanical Systems and Signal Processing, 86, pp. 17-34.

2. D. J. Ewins. 2000. "Modal Testing: Theory and Practice", Research Studies Press, UK.

3. M. A. Sutton, J. J. Orteu and H. Schreier. 2009. "Image Correlation for Shape, Motion and Deformation Measurements." Springer.

4. T. J. Beberniss, D. A. Ehrhardt. 2017. "High-speed 3D digital image correlation vibration measurement: Recent advancements and noted limitations." Mechanical Systems and Signal Processing, 86, pp. 35-48.

5. P. Poozesh, A. Sarrafi, Z. Mao, P. Avitabile, C. Niezrecki. 2017. "Feasibility of extracting operating shapes using phase-based motion magnification technique and stereo-photogrammetry.” Journal of Sound and Vibration, 407, pp. 350-366.

6. A.J. Molina-Viedma, L. Felipe-Sesé, E. López-Alba, F.A. Díaz. 2018. "3D shapes characterization using phase-based motion magnification in large structures using stereoscopic DIC.” Mechanical Systems and Signal Processing, 108, pp. 140-155.

7. A.J. Molina-Viedma, L. Felipe-Sesé, E. López-Alba, F.A. Díaz. 2018. "High frequency mode shapes characterization using Digital Image Correlation and phase-based motion magnification." Mechanical Systems and Signal Processing, 102, pp. $245-261$.

8. P. Poozesh, J. Baqersad, C. Niezrecki, P. Avitabile, E. Harvey, R. Yarala. 2017. "Large-area photogrammetry based testing of wind turbine blades." Mechanical Systems and Signal Processing, 86, pp. 98-115.

9. K. Patil, V. Srivastava, J. Baqersad. 2018. "A multi-view optical technique to obtain mode shapes of structures.” Measurement, 122, pp. 358-367.

10. J. Javh, J. Slavič, M. Boltežar. 2017. The subpixel resolution of optical-flow-based modal analysis, Mechanical Systems and Signal Processing, 88, pp. 89-99.

11. P. J. Sousa, F. Barros, P. J. Tavares, P. M. G. Moreira. 2017. "Displacement measurement and shape acquisition of an RC helicopter blade using digital image correlation.” Procedia Structural Integrity, 5, pp. 1253-1259.

12. R. Perez, G. Bartram, T. Berniss, R. Wiebe, S. M. Spottswood. 2017. "Calibration of aero-structural reduced order models using full-field experimental measurements." Mechanical Systems and Signal Processing, 86, pp. 49-65.

13. M. Flores, D. Mollenhauer, V. Runatunga, T. Beberniss, D. Rapling, M. Pankow. 2017. "High-speed 3D digital image correlation of low-velocity impacts on composite plates." Composites Part B, 131, pp. 153-164.

14. H. Leclerc, J.-N. Périé, S. Roux, F. Hild. 2009. "Integrated digital image correlation for the identification of mechanical properties." Computer Vision/Computer Graphics Collaboration Techniques 5496, pp. 161-171.

15. W. Wang, J. E. Mottershead, C. Mares, 2009a. "Vibration mode shape recognition using image processing." Journal of Sound and Vibration 326 (3-5), pp. 909-938.

16. W. Wang, J. E. Mottershead, C. Mares. 2009b. "Mode-shape recognition and finite element model updating using the Zernike moment descriptor." Mechanical Systems and Signal Processing 23 (7), pp. 2088-2112.

17. W. Wang, J. E. Mottershead, A. Ihle, T. Siebert, H. R. Schubach. 2011a. "Finite element model updating from full-field vibration measurement using digital image correlation.” Journal of Sound and Vibration 330 (8), pp. 1599-1620.

18. W. Wang, J. E. Mottershead, C. M. Sebastian, E. A. Patterson. 2011b. "Shape features and finite element model updating from full-field strain data", Int. J. Solids Struct. 48 (11-12), pp. 1644-1657.

19. W. Wang, J. E. Mottershead, C. M. Sebastian. 2011c. "Image analysis for full-field displacement/strain data: methods and applications." Appl Mech Mater, 70, pp. 39-44.

20. A. S. Patki, E. A. Patterson. 2012. "Decomposing strain maps using Fourier-Zernike shape descriptors", Exp. Mech., 52(8), pp. 1137-1149.

21. M. K. Hu. 1962. "Visual pattern recognition by moment invariants", IRE Transactions on Information Theory IT-8, pp. $179-187$.

22. F. Zernike. 1934. "Beugungstheorie des Schneidenverfahrens und Seiner Verbesserten Form, der Phasenkontrastmethode", Physica 1.

23. R. Mukundan, S. H. Ong, P. A. Lee. 2001. "Image analysis by Tchebichef moments." IEEE Transactions on Image Processing: A Publication of the IEEE Signal Processing Society 10 (9), pp. 1357-1364.

24. P. T. Yap, R. Paramesran, S. H. Ong. 2003. "Image analysis by Krawtchouk moments." IEEE Transactions on Image Processing 12(11), pp. 1367-1377.

25. I. Daubechies. 1992. "Ten Lectures on Wavelets", Society for Industrial and Applied Mathematics.

26. W. Wang, J. E. Mottershead, T. Siebert and A. Pipino. 2012. "Frequency response functions of shape features from full-field vibration measurements using digital image correlation”, Mech. Sys. Sig. Proc., 28, pp. 333-347.

27. S. Xu, X. Yang, S. Jiang. 2017. "A fast nonlocally centralized sparse representation algorithm for image denoising." Signal Processing, 131, pp. 99-112.

28. L. Liu, L. Chen, C. L. P. Chen, Y. Y. Tang, C. M. Pun. 2017. "Weighted Joint Sparse Representation for Removing Mixed Noise in Image. "IEEE Transactions on Cybernetics, 47, pp. 600-611.

29. B. Hou, K. Zhou, L, Jiao. 2018. "Adaptive Super-Resolution for Remote Sensing Images Based on Sparse Representation with Global Joint Dictionary Model.” IEEE Transactions on Geoscience and Remote Sensing, 56, pp. 2312-2327.

30. Y. Zhao, X. Sui, Q. Chen, S. Wu. 2016. "Learning-based compressed sensing for infrared image super resolution.” Infrared Physics \& Technology.

31. M. Hanif, A. Tonazzini, P. Savino, E. Salerno. 2018. "Sparse Representation Based Inpainting for the Restoration of Document Images Affected by Bleed-Through.” IWCIM Proceedings, 2, 93.

32. J. Xie, L. Xu, E. Chen. 2012. "Image denoising and inpainting with deep neural networks." In: NIPS.

33. I. Horev, O. Bryt, R. Rubinstein. 2012. "Adaptive image compression using sparse dictionaries," in Proc. 19th International Conference on Systems, Signals and Image Processing, Vienna, pp. 592-595.

34. Q. Zhang, Y. Liu, R. S. Blum, J. Han, D. Tao. 2018. "Sparse representation based multi-sensor image fusion for multi-focus and multi-modality images: A review." Information Fusion, 40, pp. 57-75. 
35. M. Aharon, M. Elad, A. M. Bruckstein. 2006. "The K-SVD: an algorithm for designing of overcomplete dictionaries for sparse representation", IEEE Transactions on Signal Processing 54, pp. 4311-4322.

36. E. W. Forgy. 1965. "Cluster analysis of multivariate data: efficiency versus interpretability of classifications." Biometrics. 21, pp. 768769.

37. S. Lesage, R. Gribonval, F. Bimbot, L. Benaroya. 2005. "Learning unions of orthonormal bases with thresholded singular value decomposition", in IEEE Int. Conf. Acoust., Speech, Signal Process.

38. D. Donoho. 2006. "Compressed sensing." IEEE Trans. Inform. Theory, vol. 52, no. 4, pp. 1289-1306.

39. M. Desbrun, M. Meyer, P. Alliez. 2002. "Intrinsic parameterizations of surface meshes." Comput. Graph. Forum 21 (3), pp. $209-218$.

40. U. Pinkall, K. Polthier. 1993. "Computing discrete minimal surfaces and their conjugates.” Exp. Math. 2 (1), pp. 15-36.

41. B. Peeters, G. De Roeck. 1999. "Referenced-based stochastic subspace identification for output-only modal analysis." Mechanical Systems and Signal Processing 13(6), pp. 855-878.

42. S. R. Ibrahim. 1977. "Random decrement technique for modal identification of structures." The AIAA Journal of Spacecraft and Rockets. 14(11).

43. S. R. Ibrahim, E. C. Mikulcik. 1977. "A method for direct identification of vibration parameters from the free response." The Shock and Vibration Bulletin. 47(4), pp. 183-98.

44. D. M. Siringoringo, Y. Fujino. 2008. "System identification of suspension bridge from ambient vibration response.” Eng. Struct. 30 (2), pp. 462-477.

45. F. Poncelet, G. Kerschen, J.-C. Golinval, D. Verhelst. 2007. "Output-only modal analysis using blind source separation techniques.", Mechanical Systems and Signal Processing, Volume 21, pp. 2335-2358.

46. E. Reynders, J. Houbrechts, G. De Roeck. 2012. "Fully automated (operational) modal analysis." Mech Syst. Signal Process 29, pp.228250.

47. C. Rainieri, G. Fabbrocino. 2014. "Operational Modal Analysis of Civil Engineering Structures: An Introduction and Guide for Applications. "Springer, New York, 322, pp. 179-185.

48. R. Brincker, L. Zhang, P. Andersen. 2000. "Modal identification from ambient responses using frequency domain decomposition", in Proceedings of the IMAC 18, International Modal Analysis Conference, pp. 625-630.

49. C. Devriendt, P. Guillaume. 2007. "The use of transmissibility measurements in output-only modal analysis", Mechanical Systems and Signal Processing 21 (7), pp. 2689-2696.

50. W. J. Yan, W. X. Ren. 2015. "An enhanced power spectral density transmissibility (EPSDT) approach for operational modal analysis: theoretical and experimental investigation.” Eng. Struct. 102, pp. 108-119.

51. B. Peeters, H. Van Der Auweraer. 2005. "PolyMax: a revolution in operational modal analysis", in: Proceedings of the IOMAC, International Operational Modal Analysis Conference, Copenhagen, Denmark.

52. S. K. Au. 2011. "Fast Bayesian FFT Method for Ambient Modal Identification with Separated Modes." J. Eng. Mech., 137(3), pp. 214-226.

53. S. K. Au. 2012a. "Fast Bayesian ambient modal identification in the frequency domain, Part I: Posterior most probable value." Mechanical Systems and Signal Processing, 26, pp. 60-75.

54. S. K. Au. 2012b. "Fast Bayesian ambient modal identification in the frequency domain, Part II: Posterior uncertainty." Mechanical Systems and Signal Processing, 26, pp. 76-90.

55. G. Oliveira, F. Magalhaes, A. Cunha and E. Caetano. 2018. "Continuous dynamic monitoring of an onshore wind turbine", Engineering Structures, 164, pp. 22-39.

56. G.-W. Chen, P. Omenzetter and S. Beskhyroun. 2017. "Operational modal analysis of an eleven-span concrete bridge subjected to weak ambient excitations, Engineering Structures, 151, pp. 839-860.

57. A. De Vivo, C. Brutti and J.L. Leofanti. 2013. "Modal shape identification of large structures exposed to wing excitation by operational modal analysis technique, Mechanical Systems and Signal Processing, 39, pp. 195-206.

58. B. Chomette and J.L. Carrou. 2015. "Operational modal analysis applied to the concert harp, Mechanical Systems and Signal Processing, 56-57, pp. 81-91

59. E. Reynders, K. Mayes, G. Lombaert and G. De Roek. 2016. "Uncertainty quantification in operational modal analysis with stochastic subspace identification: validation and applications, Mechanical Systems and Signal Processing, 66-67, pp. 13-30.

60. Y.-C. Zhu and S.-K. Au, 2018, Bayesian operational modal analysis with asynchronous data, part I: most probable value. Mechanical Systems and Signal Processing, 98, pp. 652-666.

61. Y.-C. Zhu and S.-K. Au, 2018, Bayesian operational modal analysis with asynchronous data, part II: posterior uncertainty. Mechanical Systems and Signal Processing, 98, pp. 920-935.

62. Y.-C. Zhu, Y-L. Xie and S.-K. Au, 2018, Bayesian operational modal analysis with asynchronous data, part 1: most probable value.Engineering Structures, 165, pp. 50-62.

63. S. Rizo-Patron, J. Sirohi. 2017. “Operational Modal Analysis of a Helicopter Rotor Blade Using Digital Image Correlation.” Experimental Mechcanics, 57, pp. 367-375.

64. Y.-H. Chang, W. Wang, E. A. Patterson, J.-Y. Chang, and J. E. Mottershead. 2017. "Output-only full-field modal testing." X International Conference on Structural Dynamics, EURODYN. Procedia Engineering 199, pp. 423-428.

\section{Supplementary Information: Video}

